\title{
Autoimmune Polyendocrinopathy Candidiasis Ectodermal Dystrophy: Insights into Genotype-Phenotype Correlation
}

\author{
Donatella Capalbo, ${ }^{1}$ Lucia De Martino, ${ }^{1}$ Giuliana Giardino, ${ }^{1}$ \\ Raffaella Di Mase, ${ }^{1}$ Iolanda Di Donato, ${ }^{1}$ Giancarlo Parenti, ${ }^{1}$ Pietro Vajro, ${ }^{2}$ \\ Claudio Pignata, ${ }^{1}$ and Mariacarolina Salerno ${ }^{1}$ \\ ${ }^{1}$ Department of Pediatrics, University of Naples Federico II, 80131 Naples, Italy \\ ${ }^{2}$ Department of Pediatrics, University of Salerno, 84081 Salerno, Italy \\ Correspondence should be addressed to Mariacarolina Salerno, salerno@unina.it
}

Received 15 August 2012; Revised 17 September 2012; Accepted 17 September 2012

Academic Editor: Maria L. Dufau

Copyright () 2012 Donatella Capalbo et al. This is an open access article distributed under the Creative Commons Attribution License, which permits unrestricted use, distribution, and reproduction in any medium, provided the original work is properly cited.

\begin{abstract}
Autoimmune polyendocrinopathy candidiasis ectodermal dystrophy (APECED) is a rare autosomal recessive disease, caused by mutations of a single gene named autoimmune regulator gene (AIRE) which results in a failure of $\mathrm{T}$ cell tolerance within the thymus. Chronic mucocutaneous candidiasis, chronic hypoparathyroidism, and Addison's disease are the hallmarks of the syndrome. APECED is also characterized by several autoimmune endocrine and nonendocrine manifestations, and the phenotype is often complex. Moreover, even though APECED is a monogenic disease, its clinical picture is generally dominated by a wide heterogeneity both in the severity and in the number of components even among siblings with the same AIRE genotype. The variability of its clinical expression implies that diagnosis can be challenging, and a considerable delay often occurs between the appearance of symptoms and the diagnosis. Since a prompt diagnosis is essential to prevent severe complications, clinicians should be aware of all symptoms and signs of suspicion. The aim of this paper is to give an overview on the clinical presentation and diagnostic criteria of APECED and to focus on current knowledge on genotype-phenotype correlation.
\end{abstract}

\section{Introduction}

Autoimmune polyendocrinopathy candidiasis ectodermal dystrophy (APECED) is a rare autosomal recessive disease (OMIM 240300) with a complex clinical phenotype discovered over decades. APECED is the first multiple autoimmune disease that has been shown to be caused by mutations of a single gene named autoimmune regulator gene (AIRE), which maps to $21 \mathrm{q} 22.3[1]$ and encodes a $55-\mathrm{kDa}$ protein that acts as a transcription regulator expressed in immune-related organs [2]. Immunologically, the disorder is characterized by lymphocytic infiltrate of target organs and appearance of serum autoantibodies against several defined tissue-restricted antigens, predicting or correlating with functional failure $[3,4]$. The variability of its clinical expression implies that diagnosis can often be challenging. Since a prompt diagnosis is essential to prevent severe complications, clinicians should be aware of all symptoms and signs of suspicion. Aim of this paper is to give an overview on the clinical presentation and diagnostic criteria of APECED. Moreover, attention will be paid to the current knowledge on genotype-phenotype correlation.

\section{Clinical Presentation}

APECED usually presents in childhood, but new disease components may appear even in the fifth decade of life. Clinical picture is generally characterized by a wide heterogeneity and the phenotype widely varies in the severity and in the number of components among affected subjects. This variability reflects the highly variable pattern of destructive autoimmune reactions toward different endocrine and non endocrine organs [5]. Chronic mucocutaneous candidiasis (CMC), chronic hypoparathyroidism $(\mathrm{CH})$ and Addison's disease $(\mathrm{AD})$, represent the clinical hallmark of the syndrome and the clinical diagnosis of APECED requires the presence 
of at least two of these three major components. CMC has been reported as the first sign to appear in most but not all series of reported patients. In fact, in a series of 23 Iranian Jewish patients only four had relatively mild oral, transient, candidiasis [6]. It is often followed by $\mathrm{CH}$, before the age of $10 \mathrm{yrs}$, and later by adrenal insufficiency [2]. In addition to the classic triad (CMC, $\mathrm{CH}, \mathrm{AD})$, the phenotype of APECED includes several endocrine and nonendocrine autoimmune manifestations, which in a few cases may also precede the classical triad. In fact, 10 of 91 Finnish patients had one to three other components $0.1-14$ years prior of the appearance of any of the major elements of the triad [7]. Within subjects with CMC, 21 patients had from one to six other components for $0.2-25$ years before $\mathrm{CH}$ or $\mathrm{AD}$ appeared. $\mathrm{CMC}$ is a sign of the underlying immunodeficiency, thus being different in the pathogenesis from the multiple autoimmune manifestations of APECED. It preferentially affects the oral mucosa causing a mild form of intermittent angular keilitis. More severe cases include inflammation of most of the oral mucosa, hyperplastic CMC, and atrophic form with thin mucosa and leukoplakic areas. Oesophageal and intestinal candidiasis may also occur and is characterized by abdominal pain, flatulence, and diarrhea. Patients with long-lasting oral and/or esophageal candidiasis are at increased risk of esophageal squamous cell carcinoma [7]. In the Finnish series, $10.5 \%$ of patients over 25 years of age developed squamous cell carcinoma of the oral cavity or of the esophagus [7]. This indicates that the carcinoma is not rare in these patients and, therefore, candidiasis should be aggressively treated with topical antifungals together with good oral hygiene [8]. The critical mechanisms of the increased susceptibility to CMC in APECED patients are still poorly understood although autoantibodies to cytokines seem to be implicated in the pathogenesis. Recently, a role of specific neutralizing autoantibodies against the Th17-related cytokines IL-22 and IL-17F, and the concomitant loss of Th17 and Th22 cells, has been hypothesized in the pathogenesis of the CMC [9]. On the other hand, it should be mentioned that it has been hypothesized that chronic candida infection may trigger an autoimmune disease by inducing a chronic inflammation with the persistence of high levels of cytokines.

$\mathrm{CH}$ is usually the first endocrine component. Symptoms of hypocalcemia may be vague with muscle cramps, mild paraesthesia, and clumsiness for long-time before the diagnosis is made and, sometimes, hypocalcemia may precipitate during febrile illness presenting with grand-mal type seizures. Candidate autoantigens previously linked to the development of hypoparathyroidism in APECED patients, as the calcium sensing receptor (Ca-S-R) has not been confirmed as relevant autoantigen [4, 10-13]. Recently, NALP5 (NATCH leucine-rich repeat protein 5) has been identified as the target for autoimmune attack in the parathyroid cells [14] in the context of APECED but autoantibodies against this antigen are exceptionally rare in isolated hypoparathyroidism [15].

$\mathrm{AD}$ appears most commonly between 5 and 15 years of age. Adrenal insufficiency may be asymptomatic for longtime as well as patients can report fatigue, weight loss, and increased pigmentation of mucous membrane and skin.
When unrecognized adrenal crisis may represent a fatal event. Elevated plasma ACTH and/or renin together with low cortisol are the hallmarks of the disease. However, it should be highlighted that an increase in renin levels alone may be the first biochemical abnormality of $\mathrm{AD}$, since the destructions of adrenal zona fascicualta and zona glomerulosa often are not simultaneous, the latter being the first layer targeted by the autoimmune attack [16]. The majority of patients with APECED may display autoantibodies against 21-hydroxilase even years before the clinical onset of the disease [4]. The detection of these antibodies calls for monitoring the adrenal function to prevent adrenal crisis.

The spectrum of endocrinopathies associated with APECED in addition to $\mathrm{CH}$ and AD includes hypergonadotropic hypogonadism, which is generally present only in affected females, type 1 diabetes, autoimmune thyroid diseases, and pituitary defects [17]. The occurrence of these manifestations is usually associated with a specific array of organ-specific autoantibodies that can appear quite before the overt clinical manifestation.

The presence of ectodermal abnormalities is also common. The main ectodermal manifestations in APECED are dental enamel hypoplasia, pitted nail dystrophy, and alopecia. Keratopathy, vitiligo, calcifications of the tympanic membranes, and rash with fever can also be present [7, 17]. In young patients periodic maculopapular, morbilliform, or urticarial rash usually with fever can be part of the first manifestation and in most, appears before the age of 5 years [7]. Although the pathogenesis of ectodermal dystrophies seems to be autoimmune, no specific antibodies associated have been reported to date.

Ocular manifestations are keratopathy, dry eye, sublenticular cataract, iridocyclitis, retinal detachment and optic atrophy [18]. Among these, keratopathy can be a severe complication which, in the absence of appropriate treatment, may lead to blindness [7].

Furthermore, gastrointestinal autoimmunity in APECED may lead to autoimmune gastritis, autoimmune hepatitis, and intestinal disorders with chronic diarrhea alternating with obstipation [17]. Autoimmune hepatitis can vary from mild and self-limited to severe forms requiring treatment with immunosuppressants [7] and is characterized by the presence of peculiar immunological markers such as autoantibodies against cytochrome P450IA2 (CYP IA2), CYP2A6, and aromatic L-aminoacid decarboxylase (AADC) [19-21]. Malabsorption and steatorrhea can be the result of exocrine pancreatic failure [7]. Interestingly, the intestinal endocrine cells are also the targets of autoimmune attack, and, with this regard, it has been suggested that intestinal dysfunction can also be considered to be an endocrinopathy [22]. Gastrointestinal symptoms have been associated with the presence of autoantibodies against tryptophan hydroxylase (TPHAbs) [23]. Cholelitiasis can also occur [24].

Asplenia, tubulointerstitial nephritis, obstructive lung disease, vasculitis, Sjögren's syndrome, cutaneous vasculitis, hemolytic anemia, scleroderma, metaphyseal dysplasia, and celiac disease have also been reported to be associated with APECED $[25,26]$. Autoantibodies directed against the potassium channel regulatory protein (KCNRG), found in 
epithelial cells of terminal bronchioles, have been suggested as a marker for pulmonary disease in APECED patients [27]. The autoimmune nature of renal destruction has been confirmed by examining biopsy samples and by determining antiproximal tubular autoantibodies [17, 28, 29]. Acquired asplenia, presenting in up to $20 \%$ of APECED patients [7], results in impaired immune responses to encapsulated bacteria and is a serious risk factor for developing septicemia [30]. Asplenia can be suspected on the basis of the presence of peripheral blood smears of Howell-Jolly cells. The pathogenesis of asplenia remains unknown.

Muscle disease is an additional component of APECED. Six cases have been so far described with very similar clinical features of progressive limb-girdle myopathy in the context of APECED [31]. Myopathy with axial muscle involvement may also lead to a respiratory involvement and, eventually, life-threatening respiratory failure [31].

To date, only 2 cases of encephalitis have been reported in the context of APECED one of them leading to a severe and life-threatening condition [32,33].

Life expectancy of patients with APECED depends on the severity of the disease. The overall mortality of patients with APECED varies widely on the basis of the clinical spectrum. The most dangerous autoimmune manifestations are fulminant necrotizing hepatitis, severe malabsorption, and tubulointerstitial nephritis. Suboptimal hormonal substitution or inadequate management of addisonian crisis, may also increase the mortality risk. Furthermore, patients with long-lasting oral candidiasis are at increased risk of esophageal squamous cell carcinoma [7].

Disease targeted therapy is not currently available and the treatment mainly relies on hormone replacement and caring for clinical symptoms. So far, immunosuppressive therapy has only been used for potentially fatal disease such as hepatitis, nephritis, or severe malabsorption. New examples of immunomodulatory treatment of Aire knockout mouse both targeting $\mathrm{T}$ and $\mathrm{B}$ cells $[34,35]$ lend hope that such strategies could also be useful in the future for these patients. More recently, a monoclonal antibody against B cells, Rituximab, has been successfully used to treat pulmonary disease in APECED patients, raising the hope of applying it to all patients with APECED [36].

\section{Diagnosis}

The diagnosis of APECED is primarily based on the presence of two of the three most common clinical features: CMC, $\mathrm{CH}$, and $\mathrm{AD}$. The presence of only one component is sufficient for the diagnosis if a sibling is affected. However, the early clinical picture can be dominated by one of the minor components or the presence of only one major component and, in these circumstances, APECED can be misdiagnosed. Therefore, in children the presence of a minor component should prompt to carefully investigate for other symptoms. Candidiasis in childhood (and even more in adolescence or adulthood) is often underestimated as an initial symptom of a more complex disease. In a recent survey on 24 patients affected by APECED, Mazza et al. [32] underlined the considerable delay between the appearance of the first symptoms of APECED and the time of diagnosis of the disease with a mean diagnostic delay of about 10 years.

The identification of causal genetic mutations in AIRE can confirm the diagnosis and may be helpful in those cases with atypical presentation. In about $95 \%$ of patients, two disease-causing AIRE mutations are detected [17]. Autoantibodies also constitute important diagnostic markers and may be in some cases predictive of a specific disease manifestation as detailed in Table 1 [3, 4, 13-15, 19-21, 24, 26-28, 37-43].

Recently, neutralizing autoantibodies for type 1 interferons (INF) (IFN- $\omega$ and IFN- $\alpha$ ) have been highly correlated with AIRE deficiency, regardless of the AIRE genotype, APECED features and duration. Therefore, they appear as a precious diagnostic tool to screen patients with unusual clinical manifestations of APECED instead of a more expensive and unjustified AIRE sequencing [44, 45]. In particular, AntiIFN- $\omega$ antibodies seem to appear very early in life and their presence virtually confirms the diagnosis $[45,46]$. Therefore, these autoantibodies have been recently included in the new diagnostic criteria for the diagnosis of APECED as reported by Husebye et al. [17] and shown in Table 2.

\section{Genetic Background}

As already mentioned, APECED is caused by mutations in the transcriptional regulator, AIRE. AIRE is a crucial factor in the central tolerance for the right development of selftolerance promoting clonal-deletion of self-reactive thymocytes. Within thymic medullary epithelial cells (mTECs), AIRE induces the expression of a broad repertoire of peripheral tissue antigens (PTAs) normally expressed in the periphery, eventually leading to the deletion of T-autoreactive cells $[2,47]$. Therefore, the absence of AIRE results in impaired clonal deletion of self-reactive thymocytes, which attack a variety of organs. Moreover, there is now strong evidence for AIRE expression in peripheral tissues even if these levels are significantly lower than in thymic stromal cells. The lineage of extrathymic AIRE expressing cells have been described as both myeloid [48-52] and epithelial [53] in particular in lymph node, fetal liver, and appendix tissues.

Although APECED is rare, it is relatively more frequent in some populations $(1: 9000$ in the Iranian Jews [6], $1: 25000$ in Finns $[5,54]$, and $1: 14.400$ in Sardinians [55]). It is also quite frequent in Norway $(1: 90.000)$ [56] and other regions of Italy [57]. Even though the most frequent mode of inheritance is autosomal recessive, an Italian family with APECED harbored a missense (G228W) mutation in the exon 6 in heterozygosity, indicating a dominant pattern of inheritance [58]. So far, over 70 different mutations of the AIRE gene have been documented in APECED patients [2] (Figure 1). Some different mutations have been found to be peculiar to certain populations. R257X is the most common mutation among Finnish and other European patients [59-61], 1094-1106 del113 (or 967-979 del13 bp) is the most common mutation in British [62], Irish [63], North America [64, 65], and Norwegian patients [56], and the $\mathrm{Y} 85 \mathrm{C}$ is the only mutation found among Iranian Jews [66]. In Italy APECED shows an increased prevalence in various regions, in particular in Sardinia, Apulia, and Venetian area. 
TABLE 1: Main clinical features of APECED, specific related autoantibodies (when clearly associated with clinical manifestation) and usual period of life at onset of disease.

\begin{tabular}{|c|c|c|}
\hline Clinical manifestation & Autoantibody specificities & Usual age at onset \\
\hline \multicolumn{3}{|l|}{ Classic triad } \\
\hline (i) Candidiasis & IL-17F, IL-22 [9] & Childhood \\
\hline (ii) Hypoparathyroidism & NALP5, CaSR [13-15] & Childhood \\
\hline (iii) Adrenal failure & $\mathrm{P} 450 \mathrm{c} 17, \mathrm{P} 450 \mathrm{c} 21, \mathrm{P} 450 \mathrm{scc}[4,37,42,83]$ & Childhood and adolescence \\
\hline \multicolumn{3}{|l|}{ Other endocrine disorders } \\
\hline (i) Ovarian failure & $\mathrm{P} 450 \mathrm{scc}$ and $\mathrm{P} 450 \mathrm{c} 17$ [4] & Adolescence to adulthood \\
\hline (ii) Testicular failure & TSGA10 $[84]$ & Adolescence to adulthood \\
\hline (iii) Diabetes mellitus & IA-2, GAD65 $[4,42,43]$ & Adulthood \\
\hline (iv) Hypothyroidism & TG, TPO $[38,43]$ & Childhood to adulthood \\
\hline (v) Hypopituitarism & TDRD6 [41] & Adolescence to adulthood \\
\hline \multicolumn{3}{|l|}{ Ectodermal features } \\
\hline (i) Alopecia & $\mathrm{TH}$, hair follicles $[4,85]$ & Childhood to adulthood \\
\hline (ii) Vitiligo & Melanocyte, SOX9, SOX10, AADC [39] & Childhood to adulthood \\
\hline (iii) Keratopathy & Unknown & Childhood and adolescence \\
\hline (iv) Enamel dysplasia & Unknown & Childhood \\
\hline (v) Rash with fever & Unknown & Childhood \\
\hline \multicolumn{3}{|l|}{ Gastrointestinal disorders } \\
\hline (i) Gastritis/pernicious anemia & $\mathrm{H}^{+} / \mathrm{K}^{+}$ATPase, IF $[26,43]$ & Childhood to adulthood \\
\hline (ii) Severe obstipation & TPH, HDC $[4,23,40]$ & Childhood to adulthood \\
\hline (iii) Chronic diarrhea & TPH, HDC $[4,23,40]$ & Childhood to adulthood \\
\hline (iv) Immune Hepatitis & CYP1A2, CYP2AC, AADC, TPH, HDC [3, 4, 19-21] & Childhood \\
\hline Lung manifestations & KCNRG [27] & Childhood to adulthood \\
\hline Tubulointerstitial nephritis & Unknown & Childhood \\
\hline Asplenia & Unknown & Childhood to adulthood \\
\hline
\end{tabular}

IL-17F: interleukin 17F, IL-22: interleukin 22, NALP5: NACHT leucine-rich-repeat protein 5, CaSR: calcium-sensing receptor, P450c17: Steroid 17- $\alpha$ hydroxylase, P450c21: steroid 21-hydroxylase, P450scc: side-chain cleavage enzyme, TSGA10: testis-specific gene 10 protein, IA-2: islet antigen-2, GAD65: glutamic acid decarboxylase-65, Tg: thyroglobulin, TPO: thyroid peroxidase, TDRD6: tudor domain-containing protein 6, TH: tyrosine hydroxylase, AADC: aromatic l-amino acid decarboxylase, IF: intrinsic factor, TPH: tryptophan hydroxylase, HDC: histidine decarboxylase, CYP1A2: cytochrome P450 1A2, CYP2AC: cytochrome P450 2AC, KCNRG: potassium channel-regulating protein.

TABLE 2: New diagnostic criteria for the diagnosis of APECED as reported by Husebye et al. [17].

One of the following three criteria is necessary for a definitive diagnosis:

(i) presence of at least two of the three major components: chronic mucocutaneous candidiasis, hypoparathyroidism, or adrenal Insufficiency

(ii) only one major component if a sibling is affected by APECED

(iii) disease-causing mutations in both AIRE genes

One of the following three criteria suggests a probable diagnosis:

(i) presence of one of the three major components (before 30 years of age) and at least one of the minor components

(ii) any component in the presence of anti-interferon antibodies

(iii) any component in the presence of antibodies against NALP5, AADC, TPH, or TH

AIRE: autoimmune regulator, NALP5: NACHT leucine-rich repeat protein 5, AADC: aromatic L-mino acid decarboxylase, TPH: tryptophan hydroxylase, TH: tyrosine hydroxylase.

Moreover, both in Sardinia and Apulia peculiar mutations of AIRE have been identified: the mutation R139X on exon 3 in Sardinia $[21,67]$ and the mutations W78R and Q358X on exon 2 and 9, respectively, in Apulia [68]. In the Veneto region, AIRE mutations (R257X and 979 del-13 bp on exon 6 and 8 , respectively) were different from the other
Italian regions but similar to that identified in Finnish and Anglo-saxon patients [69]. Among Sicilian patients, the typical mutation is R203X on exon 5 [70] but two novel mutations have been recently identified (S107C and Q108fs on exon 3) [71]. Although not showing a typical gene mutation, the patients from Campania exhibit a high frequency of 
(a)

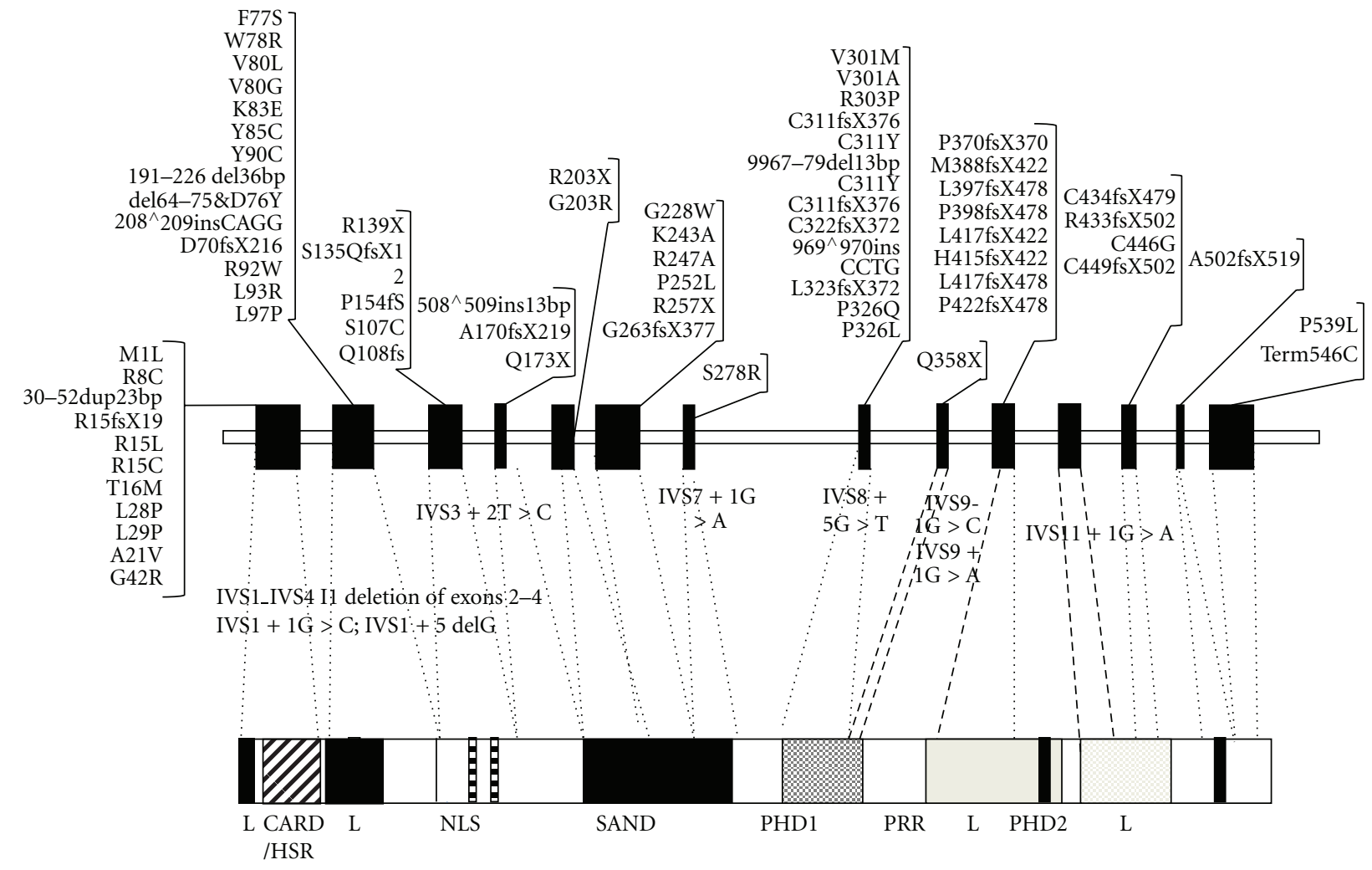

FIGURE 1: AIRE gene mutations (a) and functional domains of corresponding protein (b). Modified by Meloni et al [68].

mutations in the exon/intron 1 junction [57]. No AIRE gene mutation specific to Calabria has been found in patients with the disease [72].

\section{Genotype-Phenotype Correlation}

APECED is characterized by a wide variability of the clinical expression. In the largest reported series of 91 Finnish patients, a wide variation of the clinical phenotype and of the clinical course of APECED has been confirmed [7]. Later on, many other authors confirmed this phenotypic heterogeneity among several populations $[54,55,57,60,63,65,69$, $71,73]$. In the majority of these studies, no genotype-phenotype correlation has been found. However, several observations suggest that a genotype-phenotype correlation may exist. In fact, the Iranian Jewish patients' phenotype and genotype are peculiar of their population and differ markedly from others [6]. There is also evidence for a different sex prevalence of hypergonadotropic hypogonadism [7] and hypoparathyroidism [74]. Moreover, the G228W mutation has been associated with a peculiar phenotype. In fact, this mutation seems to elicit an unusually high risk of autoimmune thyroiditis (AT), while showing a lower penetrance for APECED [58].

Noteworthy, the clinical expression of the disease can be widely different even between siblings carrying the same mutation [75]. Such heterogeneity strongly suggests that disease-modifying genes, environmental factors, as well as immune system dynamics may play a role in modulating clinical expression of the syndrome.

Recent studies revealed effects of additional genetic loci, in particular the human leukocyte antigen (HLA) complex on certain disease manifestations of APECED [76]. Associations with specific HLA haplotypes have been found for components like alopecia, $\mathrm{AD}$, and type 1 diabetes in patients with APECED [76, 77]. These haplotypes are those associated with the common, non-APECED-related forms of that specific disorder. However, only a weak association has been observed between the HLA type and autoantibody specificities in APECED patients, suggesting that in APECED the HLA alleles do not have a strong influence on autoantibody formation per se [76].

Along with the central tolerance network, which is primarily involved in pathogenesis of APECED, several other peripheral mechanisms are capable of contributing to the control and regulation of the immune system [78]. These factors are involved in the maintenance of the homeostasis of peripheral tolerance of residual autoreactive clones, which escape negative selection within the thymus and play a significant role in preventing or minimizing reactivity to selfantigens. The peripheral tolerance recognizes, as possible mechanisms, the induction of functional anergy with inactivation of self-reactive $\mathrm{T}$ cells, deletion of autoreactive clones by apoptosis through interaction of Fas/FasL [79], and the suppressive action of regulatory $\mathrm{T}$ lymphocytes (Treg). An additional mechanism involved in controlling reactivity to 
self engages in the periphery is natural killer (NK) cells activity. Therefore, alterations dependant on one of the peripheral tolerance mechanisms [79] could contribute to the wide variability of APECED's clinical expression. To date, there are only few studies on the functionality of these immunological tolerance mechanisms in patients with APECED. A decrease of CD4+CD25+ Tregs in both adults and children with APECED has been reported $[75,80]$. However, the reduction in circulating Tregs might also be secondary to the chronic fungal infection in these individuals and, therefore, their pathogenetic role in the disease still needs to be clarified [80]. Recently, several genetic, environmental, and molecular factors potentially implicated in the phenotypic variability of APECED have been investigated in two siblings affected with APECED. They were characterized by an extremely different phenotypic expression despite an identical AIRE (IVS1 + 1G > C; IVS1 + 5delG) [75]. In particular, the younger sister had a mild form of the syndrome while the older male developed a severe phenotype exhibiting an accelerated phase involving parathyroid, thyroid, oral mucosa, skin, liver, adrenal glands, bowel, and stomach culminating in a life-threatening posterior encephalopathy syndrome (PRES) [75]. PRES is a neurological condition characterized by acute encephalopathy with specific radiological findings, rarely reported in children [81]. The pathogenesis is still unclear, however there is evidence relating PRES with autoimmune diseases or use or use of immunosuppressants [57].

The exposure to infectious agents as rubella, Epstein Barr virus, cytomegalovirus, toxoplasma, varicella zoster virus, parvovirus B19, herpes simplex virus, and parainfluenza virus was ruled out as trigger factor [82]. Mechanisms of peripheral tolerance (Fas-induced apoptosis, number of TCD4+CD25+ regulatory cells, and natural killer activity) and HLA haplotype were compared in the two sibs and any significant difference was found. However, the chance is large that the two sibs differ in their genetic makeup and this could explain the difference in clinical course despite the same AIRE mutation. As for other Mendelian disorders, the interplay between multiple genetic, epigenetic, and environmental factors certainly play a role.

\section{Conclusions}

APECED is a rare, complex, autoimmune disease. Diagnosis of APECED can be challenging and, although symptoms usually appear during the childhood, the diagnosis can be delayed up to the second decade of life. The reasons of difficulty in early recognition of these patients also rely on the heterogeneity of clinical spectrum which implies that genetic and environmental factors, other than AIRE, modulate the clinical expression. Moreover, it should be mentioned that the timing of the appearance of the individual disorders profoundly varies during the childhood, thus implying that most patients may develop new disease components in the 3 rd-5th decade of life. A better understanding of these factors involved in the clinical expression of the disease could certainly improve current knowledge on the pathogenesis of APECED and help in identifying novel therapeutic targets.

\section{Conflict of Interests}

The authors declare that there is no conflict of interests.

\section{References}

[1] Finnish-German APECED Consortium, "An autoimmune disease, APECED, caused by mutations in a novel gene featuring two PHD-type zinc-finger domains," Nature Genetics, vol. 17, no. 4, pp. 399-403, 1997.

[2] D. Mathis and C. Benoist, "Aire," Annual Review of Immunology, vol. 27, pp. 287-312, 2009.

[3] E. S. Husebye, G. Gebre-Medhin, T. Tuomi et al., "Autoantibodies against, aromatic L-amino acid decarboxylase in autoimmune polyendocrine syndrome type I," The Journal of Clinical Endocrinology and Metabolism, vol. 82, no. 1, pp. 147-150, 1997.

[4] A. Söderbergh, A. G. Myhre, O. Ekwall et al., "Prevalence and clinical associations of 10 defined autoantibodies in autoimmune polyendocrine syndrome type I," The Journal of Clinical Endocrinology and Metabolism, vol. 89, no. 2, pp. 557562, 2004.

[5] P. Ahonen, S. Myllarniemi, I. Sipila, and J. Perheentupa, "Clinical variation of autoimmune polyendocrinopathy-candidiasis-ectodermal dystrophy (APECED) in a series of 68 patients," The New England Journal of Medicine, vol. 322, no. 26, pp. 1829-1836, 1990.

[6] J. Zlotogora and M. S. Shapiro, "Polyglandular autoimmune syndrome type I among Iranian Jews," Journal of Medical Genetics, vol. 29, no. 11, pp. 824-826, 1992.

[7] J. Perheentupa, "Autoimmune polyendocrinopathy-candidiasis-ectodermal dystrophy," The Journal of Clinical Endocrinology and Metabolism, vol. 91, no. 8, pp. 2843-2850, 2006.

[8] R. Rautemaa, J. Hietanen, S. Niissalo, S. Pirinen, and J. Perheentupa, "Oral and oesophageal squamous cell carcinoma-a complication or component of autoimmune polyendocrinopathy-candidiasis-ectodermal dystrophy (APECED, APS-I)," Oral Oncology, vol. 43, no. 6, pp. 607-613, 2007.

[9] K. Kisand, A. S. Bøe Wolff, K. T. Podkrajšek et al., "Chronic mucocutaneous candidiasis in APECED or thymoma patients correlates with autoimmunity to Th17-associated cytokines," The Journal of Experimental Medicine, vol. 207, no. 2, pp. 299308, 2010.

[10] E. M. Brown, P. M. Vassilev, and S. C. Hebert, "Calcium ions as extracellular messengers," Cell, vol. 83, no. 5, pp. 679-682, 1995.

[11] Y. Li, Y. H. Song, N. Rais et al., "Autoantibodies to the extracellular domain of the calcium sensing receptor in patients with acquired hypoparathyroidism," The Journal of Clinical Investigation, vol. 97, no. 4, pp. 910-914, 1996.

[12] M. Gylling, E. Kääriäinen, R. Väisänen et al., “The hypoparathyroidism of autoimmune polyendocrinopathy-candidiasisectodermal dystrophy protective effect of male sex," The Journal of Clinical Endocrinology and Metabolism, vol. 88, no. 10, pp. 4602-4608, 2003.

[13] N. G. Gavalas, E. H. Kemp, K. J. E. Krohn, E. M. Brown, P. F. Watson, and A. P. Weetman, "The calcium-sensing receptor is a target of autoantibodies in patients with autoimmune polyendocrine syndrome type 1," The Journal of Clinical Endocrinology and Metabolism, vol. 92, no. 6, pp. 2107-2114, 2007.

[14] M. Alimohammadi, P. Björklund, A. Hallgren et al., "Autoimmune polyendocrine syndrome type 1 and NALP5, a parathyroid autoantigen," The New England Journal of Medicine, vol. 358 , no. 10 , pp. $1018-1028,2008$. 
[15] N. Tomar, E. Kaushal, M. Das et al., "Prevalence and significance of NALP5 autoantibodies in patients with idiopathic hypoparathyroidism," The Journal of Clinical Endocrinology and Metabolism, vol. 97, no. 4, pp. 1219-1226, 2012.

[16] C. Betterle, C. Dal Pra, F. Mantero, and R. Zanchetta, "Autoimmune adrenal insufficiency and autoimmune polyendocrine syndromes: autoantibodies, autoantigens, and their applicability in diagnosis and disease prediction," Endocrine Reviews, vol. 23, no. 3, pp. 327-364, 2002.

[17] E. S. Husebye, J. Perheentupa, R. Rautemaa, and O. Kämpe, "Clinical manifestations and management of patients with autoimmune polyendocrine syndrome type i," Journal of Internal Medicine, vol. 265, no. 5, pp. 514-529, 2009.

[18] L. Merenmies and A. Tarkkanen, "Chronic bilateral keratitis in autoimmune polyendocrinopathy-candidiadis-ectodermal dystrophy (APECED)," Acta Ophthalmologica Scandinavica, vol. 78, no. 5, pp. 532-535, 2000.

[19] M. G. Clemente, P. Obermayer-Straub, A. Meloni et al., "Cytochrome P450 1A2 is a hepatic autoantigen in autoimmune polyglandular syndrome type 1," The Journal of Clinical Endocrinology and Metabolism, vol. 82, no. 5, pp. 1353-1361, 1997.

[20] G. Gebre-Medhin, E. S. Husebye, J. Gustafsson et al., "Cytochrome P450IA2 and aromatic L-amino acid decarboxylase are hepatic autoantigens in autoimmune polyendocrine syndrome type I," FEBS Letters, vol. 412, no. 3, pp. 439-445, 1997.

[21] M. G. Clemente, A. Meloni, P. Obermayer-Straub, F. Frau, M. P. Manns, and S. De Virgiliis, "Two cytochromes P450 are major hepatocellular autoantigens in autoimmune polyglandular syndrome type 1," Gastroenterology, vol. 114, no. 2, pp. 324-328, 1998.

[22] R. Gianani and G. S. Eisenbarth, "Autoimmunity to gastrointestinal endocrine cells in autoimmune polyendocrine syndrome type i," The Journal of Clinical Endocrinology and Metabolism, vol. 88, no. 4, pp. 1442-1444, 2003.

[23] O. Ekwall, H. Hedstrand, L. Grimelius et al., "Identification of tryptophan hydroxylase as an intestinal autoantigen," The Lancet, vol. 352, no. 9124, pp. 279-283, 1998.

[24] T. C. Friedman, P. M. Thomas, T. A. Fleisher et al., "Frequent occurrence of asplenism and cholelithiasis in patients with autoimmune polyglandular disease type I," American Journal of Medicine, vol. 91, no. 6, pp. 625-630, 1991.

[25] E. M. Orlova, A. M. Bukina, E. S. Kuznetsova et al., "Autoimmune polyglandular syndrome type 1 in Russian patients: clinical variants and autoimmune regulator mutations," Hormone Research in Paediatrics, vol. 73, no. 6, pp. 449-457, 2010.

[26] C. Betterle, N. A. Greggio, and M. Volpato, "Clinical review 93: autoimmune polyglandular syndrome type 1," The Journal of Clinical Endocrinology and Metabolism, vol. 83, no. 4, pp. 1049-1055, 1998.

[27] M. Alimohammadi, N. Dubois, F. Sköldberg et al., "Pulmonary autoimmunity as a feature of autoimmune polyendocrine syndrome type 1 and identification of KCNRG as a bronchial autoantigen," Proceedings of the National Academy of Sciences of the United States of America, vol. 106, no. 11, pp. 4396-4401, 2009.

[28] M. Al-Owain, N. Kaya, H. Al-Zaidan et al., "Renal failure associated with APECED and terminal 4q deletion: Evidence of autoimmune nephropathy," Clinical and Developmental Immunology, vol. 2010, Article ID 586342, 7 pages, 2010.

[29] T. Ulinski, L. Perrin, M. Morris et al., "Autoimmune polyendocrinopathy-candidiasis-ectodermal dystrophy syndrome with renal failure: impact of posttransplant immunosuppression on disease activity," The Journal of Clinical Endocrinology and Metabolism, vol. 91, no. 1, pp. 192-195, 2006.
[30] A. Di Sabatino, R. Carsetti, and G. R. Corazza, "Post-splenectomy and hyposplenic states," The Lancet, vol. 378, no. 9785, pp. 86-97, 2011.

[31] M. Watanabe, H. Ochi, H. Arahata et al., "Myopathy in autoimmune polyendocrinopathycandidiasis-ectodermal dystrophy," Muscle Nerve, vol. 45, no. 6, pp. 904-908, 2012.

[32] C. Mazza, F. Buzi, F. Ortolani et al., "Clinical heterogeneity and diagnostic delay of autoimmune polyendocrinopathycandidiasis-ectodermal dystrophy syndrome," Clinical Immunology, vol. 139, no. 1, pp. 6-11, 2011.

[33] D. Capalbo, A. Elefante, M. I. Spagnuolo et al., "Posterior reversible encephalopathy syndrome in a child during an accelerated phase of a severe APECED phenotype due to an uncommon mutation of AIRE," Clinical Endocrinology, vol. 69, no. 3, pp. 511-513, 2008.

[34] J. J. DeVoss, A. K. Shum, K. P. A. Johannes et al., "Effector mechanisms of the autoimmune syndrome in the murine model of autoimmune polyglandular syndrome type 1," Journal of Immunology, vol. 181, no. 6, pp. 4072-4079, 2008.

[35] I. Gavanescu, C. Benoist, and D. Mathis, "B cells are required for Aire-deficient mice to develop multi-organ autoinflammation: a therapeutic approach for APECED patients," Proceedings of the National Academy of Sciences of the United States of America, vol. 105, no. 35, pp. 13009-13014, 2008.

[36] J. Popler, M. Alimohammadi, O. Kämpe et al., "Autoimmune polyendocrine syndrome type 1: utility of KCNRG autoantibodies as a marker of active pulmonary disease and successful treatment with rituximab," Pediatric Pulmonology, vol. 47, no. 1, pp. 84-87, 2012.

[37] O. Winqvist, J. Gustafsson, F. Rorsman, F. A. Karlsson, and O. Kampe, "Two different cytochrome P450 enzymes are the adrenal antigens in autoimmune polyendocrine syndrome type I and Addison's disease," The Journal of Clinical Investigation, vol. 92, no. 5, pp. 2377-2385, 1993.

[38] R. Perniola, A. Falorni, M. G. Clemente, F. Forini, E. Accogli, and G. Lobreglio, "Organ-specific and non-organ-specific autoantibodies in children and young adults with autoimmune polyendocrinopathy-candidiasis-ectodermal dystrophy (APECED)," European Journal of Endocrinology, vol. 143, no. 4, pp. 497-503, 2000.

[39] H. Hedstrand, O. Ekwall, M. J. Olsson et al., "The transcription factors SOX9 and SOX10 are vitiligo autoantigens in autoimmune polyendocrine syndrome type I," The Journal of Biological Chemistry, vol. 276, no. 38, pp. 35390-35395, 2001.

[40] F. Sköldberg, G. M. Portela-Gomes, L. Grimelius et al., "Histidine decarboxylase, a pyridoxal phosphate-dependent enzyme, is an autoantigen of gastric enterochromaffin-like cells," The Journal of Clinical Endocrinology and Metabolism, vol. 88, no. 4, pp. 1445-1452, 2003.

[41] S. Bensing, S. O. Fetissov, J. Mulder et al., "Pituitary autoantibodies in autoimmune polyendocrine syndrome type 1," Proceedings of the National Academy of Sciences of the United States of America, vol. 104, no. 3, pp. 949-954, 2007.

[42] A. S. B. Wolff, B. E. V. Oftedal, K. Kisand, E. Ersvær, K. Lima, and E. S. Husebye, "Flow cytometry study of blood cell subtypes reflects autoimmune and inflammatory processes in autoimmune polyendocrine syndrome type i," Scandinavian Journal of Immunology, vol. 71, no. 6, pp. 459-467, 2010.

[43] E. Proust-Lemoine, P. Saugier-Véber, D. Lefranc et al., "Autoimmune polyendocrine syndrome type 1 in North-Western France: AIRE gene mutation specificities and severe forms needing immunosuppressive therapies," Hormone Research in Paediatrics, vol. 74, no. 4, pp. 275-284, 2010. 
[44] A. Meager, K. Visvalingam, P. Peterson et al., "Anti-interferon autoantibodies in autoimmune polyendocrinopathy syndrome type 1," PLoS Medicine, vol. 3, no. 7, Article ID e289, 2006.

[45] A. Meloni, F. Incani, D. Corda, A. Cao, and M. C. Rosatelli, "Role of PHD fingers and COOH-terminal 30 amino acids in AIRE transactivation activity," Molecular Immunology, vol. 45, no. 3, pp. 805-809, 2008.

[46] B. E. Oftedal, A. S. Bøe Wolff, E. Bratland et al., "Radioimmunoassay for autoantibodies against interferon omega; its use in the diagnosis of autoimmune polyendocrine syndrome type I," Clinical Immunology, vol. 129, no. 1, pp. 163-169, 2008.

[47] M. S. Anderson, E. S. Venanzi, L. Klein et al., "Projection of an immunological self shadow within the thymus by the aire protein," Science, vol. 298, no. 5597, pp. 1395-1401, 2002.

[48] X. Zheng, L. Yin, Y. Liu, and P. Zheng, "Expression of tissuespecific autoantigens in the hematopoietic cells leads to activation-induced cell death of autoreactive T cells in the secondary lymphoid organs," European Journal of Immunology, vol. 34, no. 11, pp. 3126-3134, 2004.

[49] F. X. Hubert, S. A. Kinkel, K. E. Webster et al., "A specific antiaire antibody reveals aire expression is restricted to medullary thymic epithelial cells and not expressed in periphery," Journal of Immunology, vol. 180, no. 6, pp. 3824-3832, 2008.

[50] N. Pöntynen, M. Strengell, N. Sillanpää et al., "Critical immunological pathways are downregulated in APECED patient dendritic cells," Journal of Molecular Medicine, vol. 86, no. 10, pp. 1139-1152, 2008.

[51] E. Suzuki, Y. Kobayashi, O. Kawano et al., "Expression of AIRE in thymocytes and peripheral lymphocytes," Autoimmunity, vol. 41, no. 2, pp. 133-139, 2008.

[52] P. L. Poliani, K. Kisand, V. Marrella et al., "Human peripheral lymphoid tissues contain autoimmune regulator-expressing dendritic cells," The American Journal of Pathology, vol. 176, no. 3, pp. 1104-1112, 2010.

[53] J. M. Gardner, J. J. DeVoss, R. S. Friedman et al., "Deletional tolerance mediated by extrathymic aire-expressing cells," Science, vol. 321, no. 5890, pp. 843-847, 2008.

[54] J. Aaltonen, P. Bjorses, L. Sandkuijl, J. Perheentupa, and L. Peltonen, "An autosomal locus causing autoimmune disease: autoimmune polyglandular disease type I assigned to chromosome 21," Nature Genetics, vol. 8, no. 1, pp. 83-87, 1994.

[55] M. C. Rosatelli, A. Meloni, A. Meloni et al., "A common mutation in Sardinian autoimmune polyendocrinopathycandidiasis-ectodermal dystrophy patients," Human Genetics, vol. 103, no. 4, pp. 428-434, 1998.

[56] A. S. B. Wolff, M. M. Erichsen, A. Meager et al., "Autoimmune polyendocrine syndrome type 1 in Norway: phenotypic variation, autoantibodies, and novel mutations in the autoimmune regulator gene," The Journal of Clinical Endocrinology and Metabolism, vol. 92, no. 2, pp. 595-603, 2007.

[57] D. Capalbo, C. Mazza, R. Giordano et al., "Molecular background and genotype-phenotype correlation in APECED patients from Campania and in their relatives," Journal of Endocrinological Investigation, vol. 35, no. 2, pp. 169-173, 2012.

[58] F. Cetani, G. Barbesino, S. Borsari et al., "A novel mutation of the autoimmune regulator gene in an Italian kindred with autoimmune polyendocrinopathy-candidiasis-ectodermal dystrophy, acting in a dominant fashion and strongly cosegregating with hypothyroid autoimmune thyroiditis," The Journal of Clinical Endocrinology and Metabolism, vol. 86, no. 10, pp. 4747-4752, 2001.
[59] K. T. Podkrajšek, N. Bratanič, C. Kržišnik, and T. Battelino, "Autoimmune regulator-1 messenger ribonucleic acid analysis in a novel intronic mutation and two additional novel AIRE gene mutations in a cohort of autoimmune polyendocrinopathy-candidiasis-ectodermal dystrophy patients," The Journal of Clinical Endocrinology and Metabolism, vol. 90, no. 8, pp. 4930-4935, 2005.

[60] B. Stolarski, E. Pronicka, L. Korniszewski et al., "Molecular background of polyendocrinopathy-candidiasis-ectodermal dystrophy syndrome in a Polish population: novel AIRE mutations and an estimate of disease prevalence," Clinical Genetics, vol. 70, no. 4, pp. 348-354, 2006.

[61] H. S. Scott, M. Heino, P. Peterson et al., "Common mutations in autoimmune polyendocrinopathy-candidiasis-ectodermal dystrophy patients of different origins," Molecular Endocrinology, vol. 12, no. 8, pp. 1112-1119, 1998.

[62] S. H. S. Pearce, T. Cheetham, H. Imrie et al., "A common and recurrent 13-bp deletion in the autoimmune regulator gene in British kindreds with autoimmune polyendocrinopathy type 1," American Journal of Human Genetics, vol. 63, no. 6, pp. 1675-1684, 1998.

[63] M. Dominguez, E. Crushell, T. Ilmarinen et al., "Autoimmune polyendocrinopathy-candidiasis-ectodermal dystrophy (APECED) in the Irish population," Journal of Pediatric Endocrinology and Metabolism, vol. 19, no. 11, pp. 1343-1352, 2006.

[64] C. Y. Wang, A. Davoodi-Semiromi, W. Huang, E. Connor, J. D. Shi, and J. X. She, "Characterization of mutations in patients with autoimmune polyglandular syndrome type 1 (APS1)," Human Genetics, vol. 103, no. 6, pp. 681-685, 1998.

[65] M. Heino, H. S. Scott, Q. Chen et al., "Mutation analyses of North American APS-1 patients," Human Mutation, vol. 13, no. 1, pp. 69-74, 1999.

[66] P. Björses, M. Halonen, J. J. Palvimo et al., "Mutations in the AIRE gene: effects on subcellular location and transactivation function of the autoimmune polyendocrinopathycandidiasis-ectodermal dystrophy protein," American Journal of Human Genetics, vol. 66, no. 2, pp. 378-392, 2000.

[67] A. Meloni, N. Willcox, A. Meager et al., "Autoimmune polyendocrine syndrome type 1: an extensive longitudinal study in sardinian patients," The Journal of Clinical Endocrinology and Metabolism, vol. 97, no. 4, pp. 1114-1124, 2012.

[68] A. Meloni, R. Perniola, V. Faà, E. Corvaglia, A. Cao, and M. C. Rosatelli, "Delineation of the molecular defects in the AIRE gene in autoimmune polyendocrinopathy-candidiasis-ectodermal dystrophy patients from Southern Italy," The Journal of Clinical Endocrinology and Metabolism, vol. 87, no. 2, pp. 841-846, 2002.

[69] S. Cervato, B. Mariniello, F. Lazzarotto et al., "Evaluation of the autoimmune regulator (AIRE) gene mutations in a cohort of Italian patients with autoimmune-polyendocrinopathycandidiasis-ectodermal- dystrophy (APECED) and in their relatives," Clinical Endocrinology, vol. 70, no. 3, pp. 421-428, 2009.

[70] C. Giordano, R. Modica, M. L. Allotta et al., "Autoimmune Polyendocrinopathy-Candidiasis-Ectodermal-Dystrophy (APECED) in Sicily: confirmation that R203X is the peculiar AIRE gene mutation," Journal of Endocrinological Investigation, vol. 35, no. 4, pp. 384-388, 2012.

[71] M. Valenzise, M. Wasniewska, S. Mirabelli et al., "Identification of two novel mutations in the first Sicilian APECED patient with no R203X mutation in AIRE gene and review of Italian APECED genotypes," Gene, vol. 499, no. 2, pp. 343346, 2012. 
[72] C. Betterle, L. Ghizzoni, A. Cassio et al., "Autoimmune-Polyendocrinopathy-Candidiasis-Ectodermal-Dystrophy (APECED) in Calabria: clinical, immunological and genetic patterns," Journal of Endocrinological Investigation. In press.

[73] T. Ishii, Y. Suzuki, N. Ando, N. Matsuo, and T. Ogata, "Novel mutations of the autoimmune regulator gene in two siblings with autoimmune polyendocrinopathy-candidiasisectodermal dystrophy," The Journal of Clinical Endocrinology and Metabolism, vol. 85, no. 8, pp. 2922-2926, 2000.

[74] M. Gylling, E. Kääriäinen, R. Väisänen et al., "The hypoparathyroidism of autoimmune polyendocrinopathy-candidiasis-ectodermal dystrophy protective effect of male sex," The Journal of Clinical Endocrinology and Metabolism, vol. 88, no. 10, pp. 4602-4608, 2003.

[75] D. Capalbo, A. Fusco, G. Aloj et al., "High intrafamilial variability in autoimmune polyendocrinopathy-candidiasis-ectodermal dystrophy: a case study," Journal of Endocrinological Investigation, vol. 35, no. 1, pp. 77-81, 2012.

[76] M. Halonen, P. Eskelin, A. G. Myhre et al., "AIRE mutations and human leukocyte antigen genotypes as determinants of the autoimmune polyendocrinopathy-candidiasis-ectodermal dystrophy phenotype," The Journal of Clinical Endocrinology and Metabolism, vol. 87, no. 6, pp. 2568-2574, 2002.

[77] M. Gylling, T. Tuomi, P. Björses et al., " $\beta$-cell autoantibodies, human leukocyte antigen II alleles, and type 1 diabetes in autoimmune polyendocrinopathy-candidiasis-ectodermal dystrophy," The Journal of Clinical Endocrinology and Metabolism, vol. 85, no. 12, pp. 4434-4440, 2000.

[78] J. A. Bluestone, "Mechanisms of tolerance," Immunological Reviews, vol. 241, no. 1, pp. 5-19, 2011.

[79] S. DeFranco, S. Bonissoni, F. Cerutti et al., "Defective function of Fas in patients with type 1 diabetes associated with other autoimmune diseases," Diabetes, vol. 50, no. 3, pp. 483-488, 2001.

[80] E. Kekäläinen, H. Tuovinen, J. Joensuu et al., "A defect of regulatory $\mathrm{T}$ cells in patients with autoimmune polyendocrinopathy-candidiasis-ectodermal dystrophy," Journal of Immunology, vol. 178, no. 2, pp. 1208-1215, 2007.

[81] J. Hinchey, C. Chaves, B. Appignani et al., "A reversible posterior leukoencephalopathy syndrome," The New England Journal of Medicine, vol. 334, no. 8, pp. 494-500, 1996.

[82] C. Pignata, M. Fiore, S. de Filippo, M. Cavalcanti, L. Gaetaniello, and I. Scotese, "Apoptosis as a mechanism of peripheral blood mononuclear cell death after measles and varicellazoster virus infections in children," Pediatric Research, vol. 43, no. 1, pp. 77-83, 1998.

[83] K. Krohn, R. Uibo, E. Aavik, P. Peterson, and Savilahti, "Identification by molecular cloning of an autoantigen associated with Addison's disease as steroid $17 \alpha$-hydroxylase," The Lancet, vol. 339, no. 8796, pp. 770-773, 1992.

[84] K. Reimand, J. Perheentupa, M. Link, K. Krohn, P. Peterson, and R. Uibo, "Testis-expressed protein TSGA10-an autoantigen in autoimmune polyendocrine syndrome type I," International Immunology, vol. 20, no. 1, pp. 39-44, 2008.

[85] H. Hedstrand, O. Ekwall, J. Haavik et al., "Identification of tyrosine hydroxylase as an autoantigen in autoimmune polyendocrine syndrome type I," Biochemical and Biophysical Research Communications, vol. 267, no. 1, pp. 456-461, 2000. 


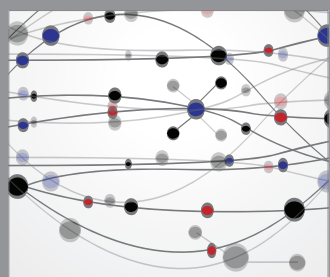

The Scientific World Journal
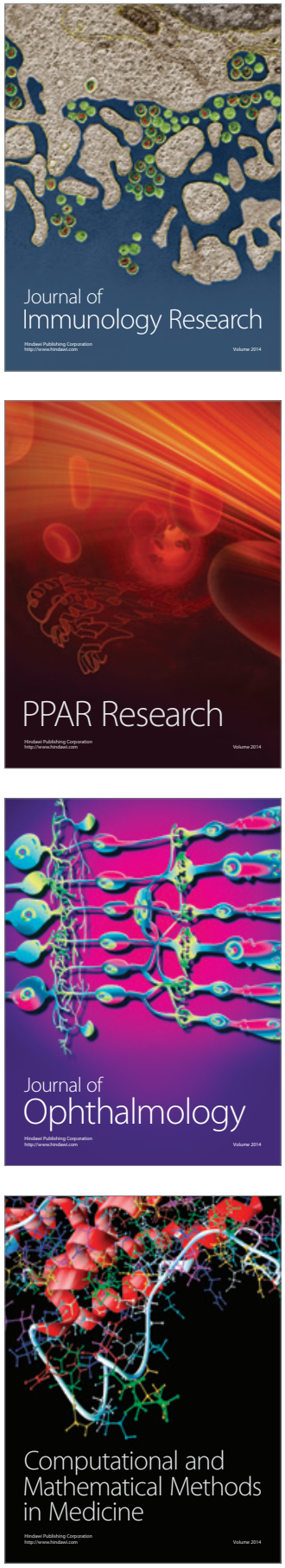

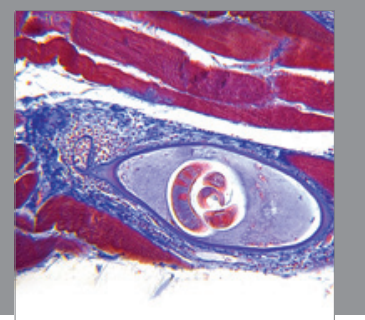

Gastroenterology

Research and Practice
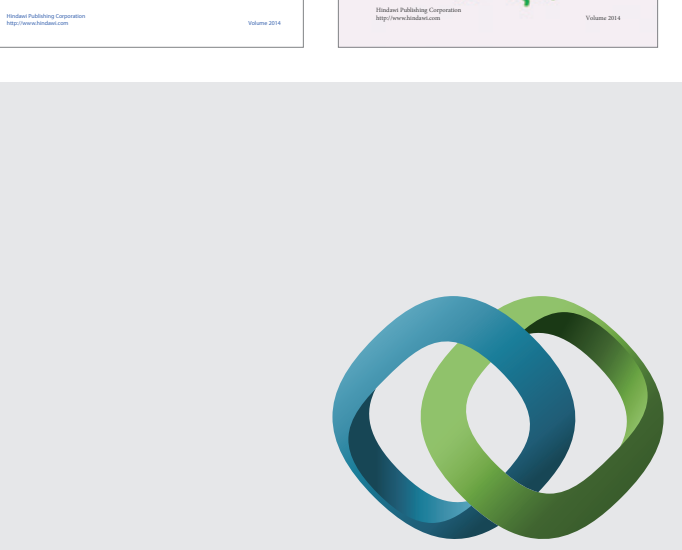

\section{Hindawi}

Submit your manuscripts at

http://www.hindawi.com
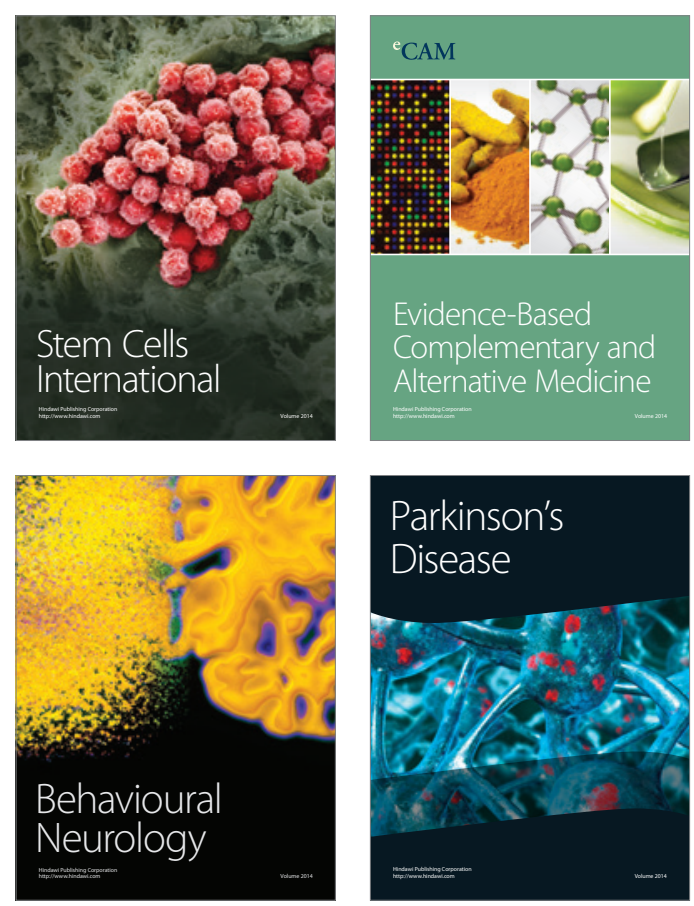

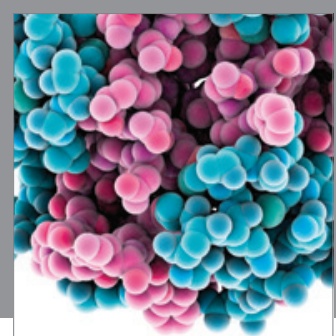

Journal of
Diabetes Research

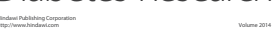

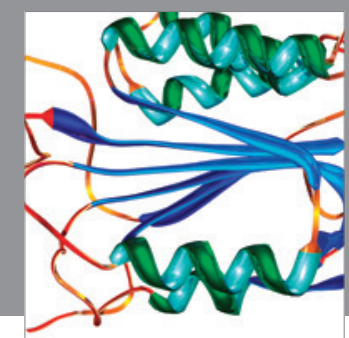

Disease Markers
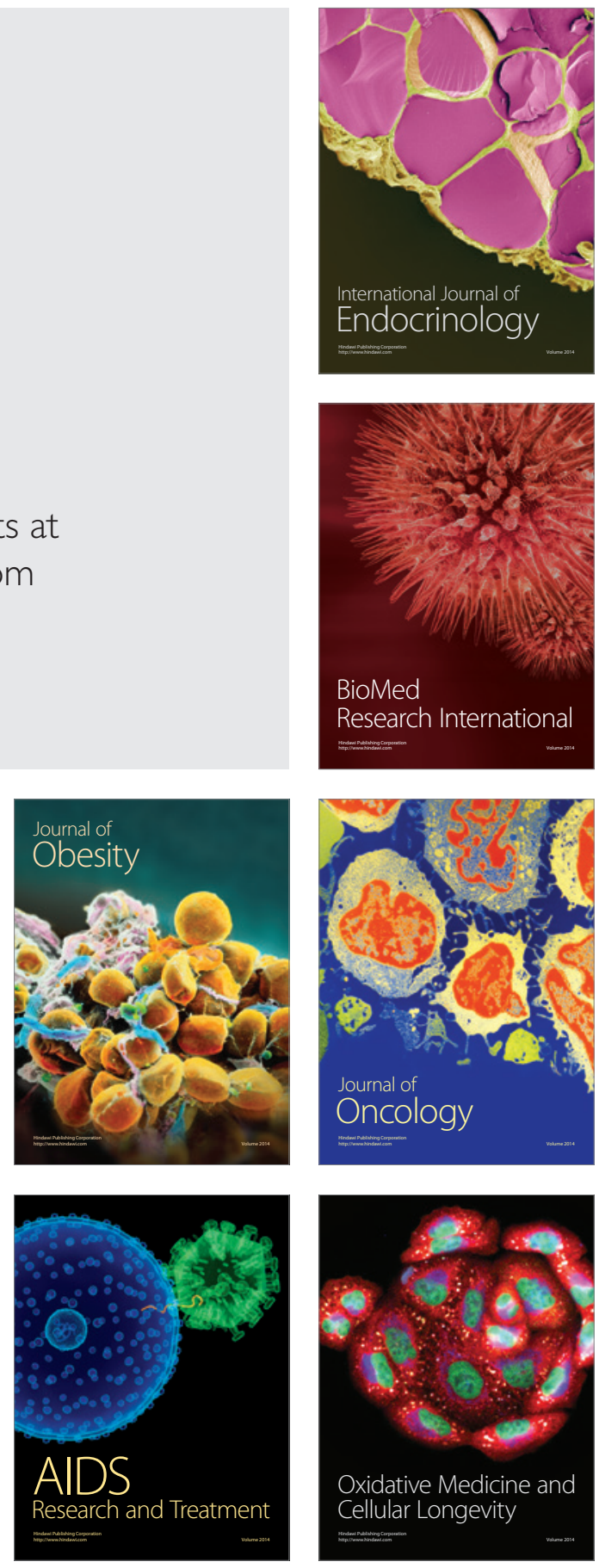